\title{
MODÉLISATION DU RISQUE DE LA NOUVEAUTÉ PERÇUE SUR LA PERCEPTION D'UNE MARQUE INNOVANTE
}

\author{
Blandine HETET ${ }^{*}$, Jean-Michel MOUTOT ${ }^{* *} \&$ Jean-Pierre MATHIEU ${ }^{* * *}$
}

\begin{abstract}
Résumé - Cet article présente une recherche sur le risque de la nouveauté perçue sur la perception des consommateurs d'une marque innovante. Basée sur une revue de la littérature dans le domaine de la perception des innovations, un modèle d'analyse intégrant différentes dimensions a été proposé. Il intègre l'analyse de l'innovativité des consommateurs, mais également la mesure des attitudes implicites qui permettent l'enrichissement de la recherche. L'implémentation d'une expérimentation sur un échantillon représentatif de la population et l'analyse des données à l'aide des équations structurelles permettront de proposer une validation du modèle.
\end{abstract}

Mots clés : risque ; innovation ; nouveauté perçue ; innovativité ; marque innovante.

\section{Introduction}

L'innovation est un concept central dans la vie des entreprises aujourd'hui. En effet, pour être concurrentielles, les entreprises doivent innover (Pauwels et al., 2004). Le marketing est familier de ce concept d'innovation car il se base sur ces éléments pour communiquer et rendre cohérente sa stratégie. Mais malgré de nombreuses études et réflexions, la plupart des nouveaux produits ne trouvent pas leur place sur le marché (Srinivasan et al., 2009). Or, les innovations n'ont de sens et d'intérêt pour les entreprises que lorsqu'elles sont adoptées par les consommateurs. Le développement et la diffusion des innovations représentent un coût important en ressources pour l'entreprise qui ne peut être compensé que par leur achat ou adoption par les consommateurs.

\footnotetext{
* Enseignant-chercheur, IDRAC Business School 11 rue des Saumonières BP 4120544300 NANTES, blandine.hetet@idracnantes.com

** Professeur associé, Centre CRM chaire ERDF Audencia École de Management 8 route de la Jonelière BP 3122244312 NANTES CEDEX 3, jmoutot@audencia.com

*** Professeur, CEPN-CNRS UMR 7234 Université Paris13, Sorbonne Paris Cité et IHEP Paris, jean-pierre.mathieu@univ-paris13.fr
} 
L'adoption et la diffusion des innovations ont été beaucoup développés dans la recherche académique (Rogers, 2003 ; Roehrich, 1993, 2004 ; Volle, 1995 ; Cestre, 1996 ; Jansson, 2011 ; Flight et al., 2011), mais peu de travaux se sont intéressés à la perception de la nouveauté par les consommateurs (Roehrich, 1993). Pour Bartels et Reinders (2011), la plupart des échecs des innovations sont dus à un manque de compréhension des attentes des consommateurs. Or, l'écart de perception de la nouveauté d'un produit entre les entreprises et les consommateurs représente un risque important dans l'adoption des innovations. Selon Ziamou et Ratneshwar (2002), la question de la nouveauté présente dans ces innovations est celle envisagée par le marketeur et non par le consommateur, car les innovations, considérées comme des nouveaux produits, sont étudiées par les marketeurs, puis proposées aux consommateurs.

Par ailleurs, les innovations sont développées par les entreprises pour bénéficier d'un avantage concurrentiel, mais également pour influencer leur capital marque (Staake et al. 2009). Quelques recherches se sont intéressées aux impacts des échecs de lancement d'innovation et de leurs effets sur la satisfaction des consommateurs (Smith and Bolton, 2002 ; Roehm et Brady, 2007 ; Andreassen, 2000). Or, l'étude de Liao et Cheng (2012) a montré que l'échec d'une innovation a une influence sur l'image de marque. De plus, Kaplan (2009) souligne l'importance des innovations dans le développement de marques fortes et l'influence de la perception des innovations sur le jugement des consommateurs d'une marque innovante. Ce n'est pas parce que l'entreprise va innover et proposer de nouveaux produits qu'elle va être perçue comme innovante : encore faut-il que ces produits soient perçus par les consommateurs comme une réelle nouveauté. Ce champ de recherche a été peu développé ces dernières années, contrairement à la capacité d'innovation des marques (Beverland et al., 2009 ; Anselmsson et Johansson, 2009). De plus, l'étude de l'influence des innovations sur la perception d'une marque innovante et sur l'attitude envers la marque n'a été que peu développée (Schmitt, 2012).

L'objectif de cet article est de montrer le risque de la nouveauté perçue sur la perception d'une marque innovante par les consommateurs. La perception des innovations par les consommateurs est un processus complexe qui peut être influencé par de nombreux éléments : la testabilité, l'avantage relatif,...(Rogers, 2003). La nouveauté perçue a été peu étudiée dans la littérature, alors qu'elle représente un risque important d'influence de la perception des consommateurs des différentes variables présentes lors de l'appréciation d'une innovation. De plus, ce risque est également présent dans l'évaluation de la marque par les consommateurs, car le fait de percevoir un produit comme nouveau peut avoir un effet sur l'attitude envers la marque, et notamment sur la perception d'une marque innovante.

Cet article présentera le cadre conceptuel de la recherche en développant les différents modèles théoriques : l'adoption et la diffusion des innovations, l'innovativité des consommateurs, la perception de la nouveauté par les consommateurs, les attitudes et l'influence des consommateurs sur les marques. Puis le contexte de la recherche sera exposé, ainsi que la problématique de recherche et le modèle proposé. Enfin, en conclusion, la méthodologie de recherche envisagée sera introduite pour ouvrir sur les implications théoriques, managériales et méthodologiques de la recherche. 


\section{Cadre conceptuel de la recherche}

\subsection{L'adoption et la diffusion des innovations}

L'adoption et la diffusion des innovations a ont beaucoup développés dans la littérature (Rogers, 2003 ; Roehrich, 1993, 2004 ; Volle, 1995 ; Cestre, 1996 ; Jansson, 2011 ; Flight et al., 2011). Rogers et Shoemaker (1971) définissent une innovation comme étant une nouvelle idée, pratique ou objet perçu comme nouveau par l'individu. Un produit n'est considéré comme nouveau que s'il est perçu par les consommateurs comme tel. La nouveauté perçue, bien que présente dans la définition de Rogers et Shoemaker (1971) n'apparait pas comme une caractéristique intrinsèque du produit influençant son processus d'adoption.

En effet, Rogers (2003) a modélisé le processus de diffusion des innovations permettant l'analyse du processus de décision des consommateurs face à un nouveau produit où il propose cinq caractéristiques intrinsèques du produit qui conditionne la rapidité d'adoption : l'avantage relatif, la compatibilité, la simplicité, la testabilité (Tan et Teo, 2000) et l'observabilité.

Ces cinq caractéristiques doivent être prises en compte de manière conjointe afin de prédire l'adoption de l'innovation. D'autres critères ont été ajoutés par certains auteurs afin de compléter et d'enrichir l'analyse de la perception des consommateurs d'une innovation. Volle (1995) ou Meuter et al. (2005) ont ajouté la notion de risque perçu de l'innovation. Défini comme «l'incertitude subjective perçue par l'individu quant à l'ensemble des pertes potentielles relatives aux attributs déterminants du choix d'un produit (bien ou service) dans une situation d'achat ou de consommation donnée» par Volle (1995), le risque perçu représente une des caractéristiques centrales de l'adoption d'une innovation avec l'avantage relatif et la compatibilité (Roehrich, 2004).

Roehrich $(1993,2004)$ ajoute la nouveauté perçue à cette conception en démontrant qu'elle est indépendante des autres caractéristiques perçues de l'innovation. Il propose l'étude approfondie de la perception de la nouveauté pour approfondir l'analyse des innovations.

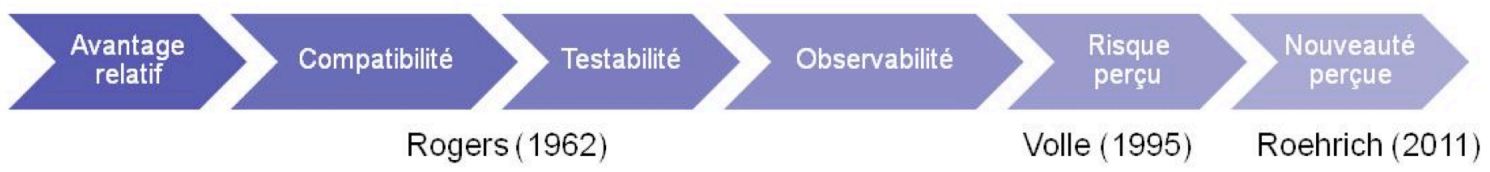

Figure 1 : variables perçues de l'innovation.

\subsection{La perception de la nouveauté}

Un produit n'est considéré comme nouveau que s'il est perçu par les consommateurs comme tel (Rogers et Shoemaker, 1971). Rogers (1995) considère la nouveauté comme étant intrinsèque à toutes les innovations et explique que «la nouveauté perçue par l'individu d'une idée détermine sa réaction» (p11). C'est également la nouveauté qui permet de distinguer l'innovation du changement (Slappendel, 1996).

Malgré la prise en compte de la nouveauté dans la définition d'une innovation (Rogers, 1995), elle reste peu étudiée. Aujourd'hui en marketing, la nouveauté des innovations est 
analysée par des critères objectifs (date de mise en marché du produit, mise en place d'une nouvelle campagne publicitaire, nouveaux attributs du produit, etc.) sans tenir compte de la nouveauté perçue par les consommateurs (Roehrich, 1993 ; Pantin-Sohier et Lancelot Miltgen, 2012 ; Mukherjee et Hoyer, 2001 ; Xuereb, 1993). C'est donc la nouveauté du point de vue de l'entreprise et non celui du consommateur (Dinnin, 2009 ; Danneels et Kleinschmidt, 2001). Selon Baregheh et al. (2009), une innovation est décrite comme quelque chose de nouveau ou unique, que ce soit un produit ou un service. Peu présente dans la littérature sur les produits nouveaux (Roehrich, 1993, 2004), Roehrich (2004) définit la nouveauté perçue comme ce qui est perçu comme étant récent et/ou différent.

Flight et al. (2011) soulignent l'importance de la perception d'une innovation par les consommateurs pour identifier les problèmes d'adoption d'une innovation. La nonperception de la nouveauté représente un risque conséquent dans la diffusion et l'adoption des innovations, mais également dans de nombreux domaines du marketing tels que l'étude du comportement et des attentes des consommateurs (Roehrich, 1993) ou la construction des marques. En effet, la nouveauté perçue peut être un critère de choix pour certains consommateurs (Haines, 1966) et détermine la réaction des consommateurs face au produit nouveau (Rogers, 2003). L'étude de la nouveauté perçue représente ainsi un avantage stratégique pour les marketeurs qui pourront développer les éléments qui différencient le produit des autres et le rendent unique aux yeux des consommateurs.

Wells et al. (2010) précisent que le degré de perception de la nouveauté d'une innovation, c'est à dire le fait qu'elle représente une alternative nouvelle par rapport à l'existant, varie selon les individus, mais constitue un élément pouvant prédire l'adoption de cette innovation. Il est donc nécessaire d'analyser la nouveauté perçue mais également l'innovativité des consommateurs. Ces deux concepts sont complémentaires et s'influencent mutuellement (Oslund, 1974).

\subsection{L'innovativité}

Chaque individu évalue un produit nouveau de manière différente et a une perception propre des innovations (Wells et al., 2010). Le terme innovativité est utilisé de manière différente dans la littérature : il peut être lié à la capacité d'innovation d'une marque ou d'un individu, mais également à la capacité d'un individu à adopter des produits nouveaux (Cestre, 1996). C'est dans cette dernière perspective que nous utiliserons ce terme. L'innovativité cherche à expliquer, par des traits de personnalité, pourquoi certains individus adoptent une innovation dès sa sortie, alors que d'autres ne l'adoptent que plus tard ou pas du tout (Rogers et Shoemaker, 1971 ; Zhuang, 1995). L'innovativité et la nouveauté perçue sont liées dans le processus d'adoption des innovations mais se distinguent car l'innovativité désigne la capacité à adopter des produits nouveaux et non la perception de la nouveauté des innovations.

De nombreuses recherches académiques ont porté sur la tendance à adopter une innovation : 1'innovativité (Bagozzi et Foxall, 1996 ; Le Louarn, 1997 ; Wood et Swait, 2002 ; Roehrich, 2004). Mais selon Tellis et al. (2009), il n'existe pas de consensus sur la mesure de l'innovativité et de nombreuses typologies et de mesures de l'innovativité ont été proposées (Roherich, 2004 ; Steenkamp et al., 1999 ; Raju, 1980 ; Venkatraman et Price, 1990 ; Hirschman, 1980 ; Midgley et Dowling, 1978). Vandecasteele et Geuens (2010) ont proposé de différencier quatre dimensions de l'innovativité à l'aide d'une échelle de mesure des 
motivations poussant les individus à acheter ou utiliser une innovation. Ils différencient quatre dimensions : fonctionnelle, hédoniste, sociale et cognitive. C'est la motivation à adopter un comportement innovateur qui est étudiée, et non pas un trait de personnalité ou les caractéristiques propres du produit. Le tableau 1 présente les quatre dimensions de l'innovativité motivationnelles telles que définies par Vandecasteele et Geuens (2010).

Vandecasteele et Geuens (2010) définissent l'innovativité comme l'ensemble des motivations incitant les individus à adopter un produit nouveau. Les auteurs distinguent quatre dimensions de l'innovativité : fonctionnelle, hédoniste, sociale et cognitive. La conception motivationnelle de l'innovativité permet donc de distinguer quatre dimensions motivationnelles, présentes chez tous les individus mais néanmoins différemment. Chaque dimension a un impact propre sur les intentions d'achats des nouveaux produits.

\begin{tabular}{|c|c|}
\hline Type d'innovativité & Définition \\
\hline Fonctionnelle & $\begin{array}{l}\text { Tendance à adopter une innovation pour ses performances } \\
\text { fonctionnelle et son utilité. }\end{array}$ \\
\hline Cognitive & $\begin{array}{l}\text { Tendance à adopter une innovation pour sa capacité à stimuler la } \\
\text { réflexion et à favoriser l'apprentissage. }\end{array}$ \\
\hline Hédoniste & $\begin{array}{c}\text { Tendance à adopter une innovation pour sa capacité à stimuler la } \\
\text { dimension affective et sensorielle des individus. }\end{array}$ \\
\hline Sociale & $\begin{array}{l}\text { Tendance à adopter une innovation pour se différencier des autres, être } \\
\text { unique et renforcer son statut social. }\end{array}$ \\
\hline
\end{tabular}

Tableau 1 : Typologie de l'innovativité (Vandecasteele et Geuens, 2010).

Chaque dimension de l'innovativité aura un impact différent sur les attitudes mais également la perception de la nouveauté par les individus.

\subsection{L'attitude}

L'attitude est une notion issue de la psychologie qui est définie comme une association stockée en mémoire entre un objet et une évaluation (Fazio et al., 1982). Elle permet de faire le lien entre un objet et l'évaluation ou le jugement émis par un individu. L'attitude des consommateurs envers des produits ou objets a une forte valeur prédictive sur les comportements (Perugini et al., 2010), notamment dans le cas d'innovations (Steyer et Zimmermann, 2004), dans la prise de décision (Arcuri et al., 2008 ; Bodenhausen et Todd, 2010 ; Fazio, 2002), le choix d'une marque (Courbet et Fourquet-Courbet, 2005) et les comportements d'achat et d'usage (Madhavaram et Appan, 2010 ; Maison et al., 2004 ; Wänke et al., 2002).

Les notions d'attitude et de cognition sociale implicite issues de la psychologie (Nosek, 2007 ; Greenwald et Banaji, 1995) ont été introduites en marketing (Ackermann, 2010 ; Ackermann et Mathieu, 2012 ; Trendel et Warlop, 2005 ; Brunel et al., 2004) car l'étude partielle des attitudes représente un risque, notamment dans l'analyse des innovations (Hetet et al., 2013 ; Hetet et Moutot, 2012). L'étude des attitudes explicites et implicites sont complémentaires et permettent de recueillir des informations différentes sur le même objet, ce qui permet de réduire les biais associés à la réflexion (désirabilité sociale, effet de demande,...) (Nosek, 2007 ; Greenwald et Krieger, 2006).

La mesure des attitudes implicites qui utilise des protocoles indirects de recueil des données (Fazio et Olson, 2003) permettent d'enrichir la compréhension du consommateur 
car une partie importante des comportements est déterminée par des processus cognitifs en dehors de la conscience (Brunel et al., 2004 ; Maison et al., 2004).

L'étude des attitudes explicites et implicites de la marque et de l'innovation va permettre d'approfondir la compréhension de la perception et du jugement des consommateurs., mais également de proposer un modèle complet du risque que représente la nouveauté perçue sur la perception d'une marque innovante.

\subsection{Lien entre marque et innovation : vers une marque innovante}

Kaplan (2009) souligne l'importance des innovations dans le développement de marques fortes. Les marques influencent les innovations qu'elles portent, mais l'inverse est également présent. Les innovations vont alors influencer la marque, et notamment la perception de la marque par les consommateurs. L'influence de la perception des innovations sur le jugement des consommateurs d'une marque innovante, est un champ de recherche qui a été peu développé (Schmitt, 2012 ; Barone et Jewell, 2013). On entend par marque innovante une marque qui est perçue comme ayant une capacité d'innovation par les consommateurs. Il s'agit d'un jugement qui n'est pas basé sur des éléments objectifs de l'entreprise. Par ailleurs, les consommateurs peuvent percevoir une marque comme innovante indépendamment des produits qu'elle propose, de par ses pratiques managériales par exemple. Ils peuvent également percevoir la nouveauté d'un produit sans pour autant juger la marque comme innovante.

Quelques recherches se sont intéressées aux impacts des échecs de lancement d'innovation et de leurs effets sur la satisfaction des consommateurs (Smith et Bolton, 2002 ; Roehm et Brady, 2007 ; Andreassen, 2000). L'échec d'une innovation a une influence sur l'image de marque (Liao et Cheng, 2012 ; Smith et Bolton, 2002 ; Roehm et Brady, 2007 ; Andreassen, 2000), mais les recherches n'ont pas développé les variables à l'origine de ces influences, préférant étudier la capacité d'innovation des marques (Beverland et al., 2009 ; Anselmsson et Johansson, 2009).

\section{Problématique et questions de recherche}

Dans le cadre de cet article, nous proposons de mettre en évidence le risque de la nouveauté perçue sur la perception par les consommateurs d'une marque innovante. Nous avons vu que le concept de nouveauté perçue était peu étudié dans la littérature et que son influence sur la perception des innovations est pourtant très importante (Roehrich, 1993, 2004). De plus, l'influence des innovations sur les marques est présente et a été étudiée dans le cadre d'échec de diffusion d'innovation (Smith et Bolton, 2002 ; Roehm et Brady, 2007 ; Andreassen, 2000), mais le concept de "marque innovante » et ses caractéristiques reste à définir. Enfin, la perception des innovations dépend également de la manière dont les consommateurs vont analyser leurs caractéristiques à travers leurs traits de personnalité. Il est donc important de prendre en compte la notion d'innovativité (Wells et al., 2010).

Nous pensons que les différentes dimensions de l'innovativité vont avoir une influence sur la perception de la nouveauté par les consommateurs. Celle-ci influencera l'attitude (explicite et implicite) des consommateurs à l'égard de l'innovation et leur perception d'une marque innovante. 
Nous proposons de traiter cette problématique sous la forme de deux questions de recherche associées au modèle présenté :

Q1 : Comment les quatre dimensions de l'innovativité influencent-elles la perception de la nouveauté et quelle en est l'influence sur les variables perçues d'une innovation?

Q2 : Quel est le rôle de la nouveauté perçue d'une innovation sur la perception d'une marque innovante?

\section{Modélisation du risque de la nouveauté perçue}

Suite à la définition du cadre théorique de la recherche, nous avons mis en évidence la place centrale de la nouveauté perçue dans le processus de perception et d'évaluation des innovations par les consommateurs. Afin de répondre à chaque question de recherche, le modèle est divisé en deux parties :

La première partie du modèle repose sur la question de recherche suivante :

Q1 : Comment les quatre dimensions de l'innovativité influencent-elles la perception de la nouveauté et quelle en est l'influence sur les variables perçues d'une innovation?

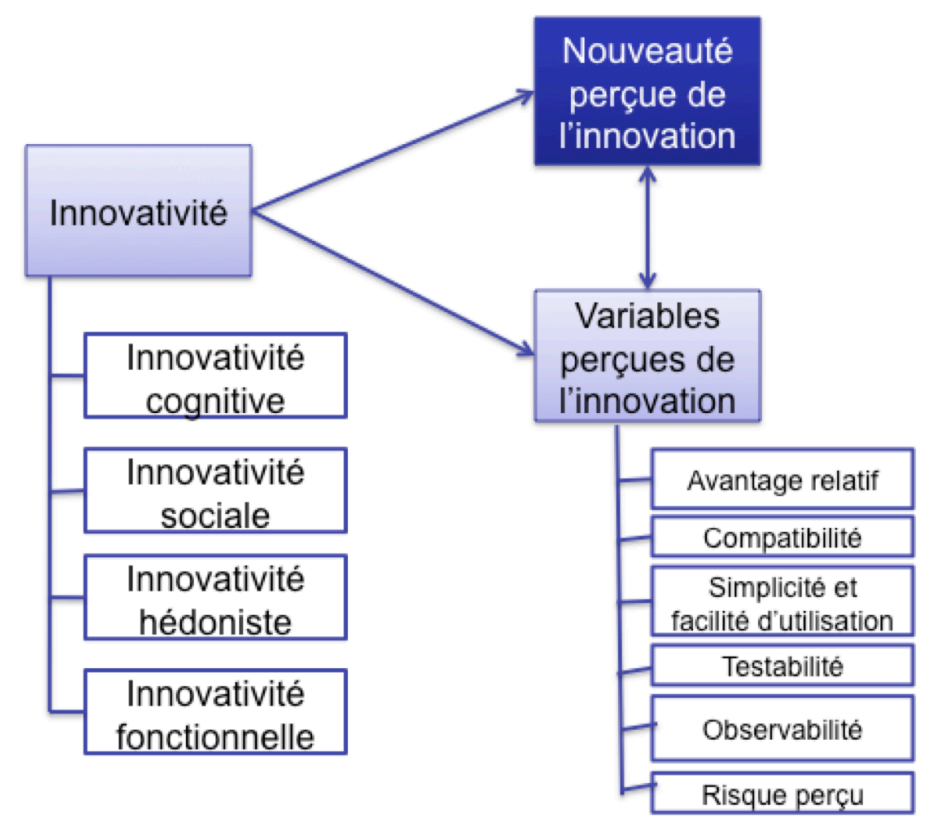

Figure 2 : Typologie d'innovativité et nouveauté perçue.

Il s'agit de comprendre comment les quatre dimensions de l'innovativité influencent la nouveauté perçue et les variables perçues de l'innovation. L'innovativité est un processus individuel qui va impacter la perception des personnes évaluant l'innovation. Chaque dimension de l'innovativité exercera une influence différente (Vandecasteele et Geuens, 2010) sur la perception de la nouveauté et sur l'importance accordée à chacune des variables perçues définies par Rogers (2003) et enrichies du risque perçu (Volle, 1995). De plus, la nouveauté perçue, bien qu'indépendante des variables perçues d'une innovation (Roehrich, 1993) sera en relation avec celles-ci. Cette première partie du modèle permettra de comprendre et de distinguer le rôle de chacune des variables présentées, et plus particulièrement celui de la nouveauté perçue. 
La seconde partie du modèle repose sur la question de recherche suivante :

Q2 : Quel est le rôle de la nouveauté perçue d'une innovation sur la perception d'une marque innovante?

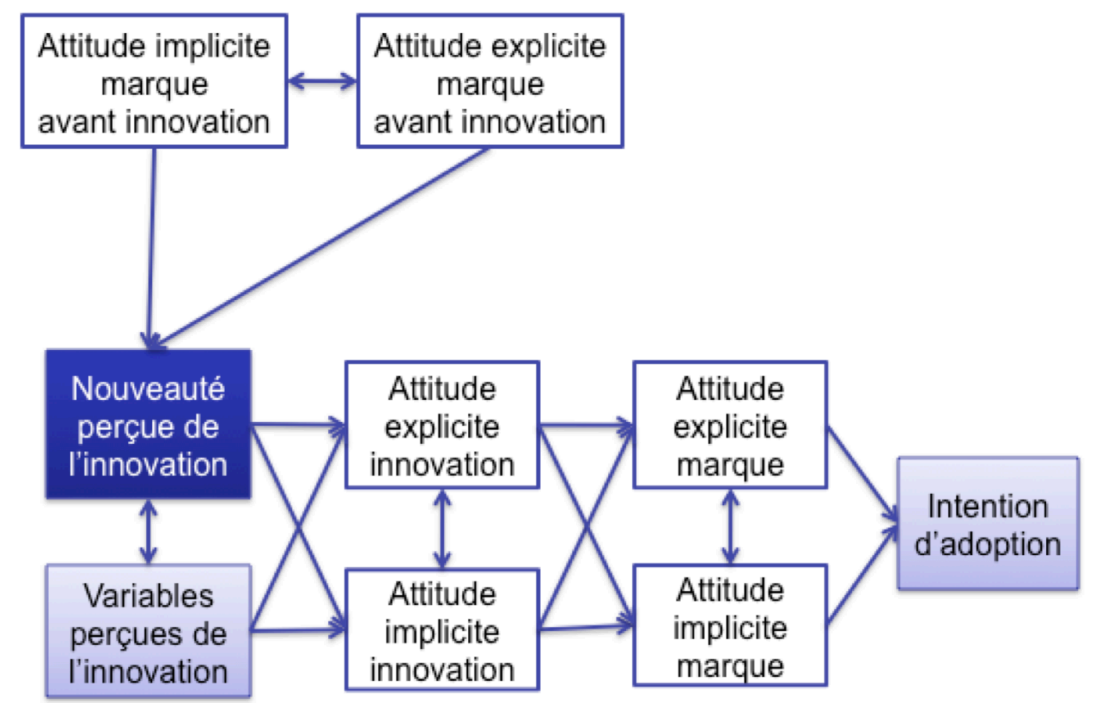

Figure 3 : Marque innovante et nouveauté perçue.

La nouveauté perçue, au centre de notre modèle, va avoir une influence sur l'attitude implicite et explicite des consommateurs sur une innovation. Afin de mesurer l'influence de la nouveauté perçue sur la perception des consommateurs, il est nécessaire de comparer leur attitude envers la marque avant la présentation de l'innovation, pour établir une base de comparaison. Ainsi, nous pouvons comparer l'attitude initiale envers la marque, sans que les consommateurs n'aient connaissance de l'innovation, et l'attitude envers la marque après la présentation de l'innovation. Les différences observées nous permettrons d'évaluer l'influence de l'attitude envers l'innovation, mais également de la nouveauté perçue sur la perception des consommateurs de la marque, et notamment de son caractère innovant.

La mesure complémentaire des attitudes explicites et implicites permettra d'enrichir la compréhension des attitudes des consommateurs et d'évaluer leur perception de la marque comme innovante. Cette utilisation permet notamment de prévenir l'effet de demande, très présent dans le cas d'innovation pour laquelle le consommateur n'a pas encore de représentation en mémoire.

Enfin, la mesure de l'intention d'adoption de l'innovation permettra de prédire les comportements des consommateurs et de qualifier l'influence de la nouveauté perçue sur les attitudes, mais également sur les comportements.

Ces deux questions de recherche nous permettront de proposer le modèle global suivant: 


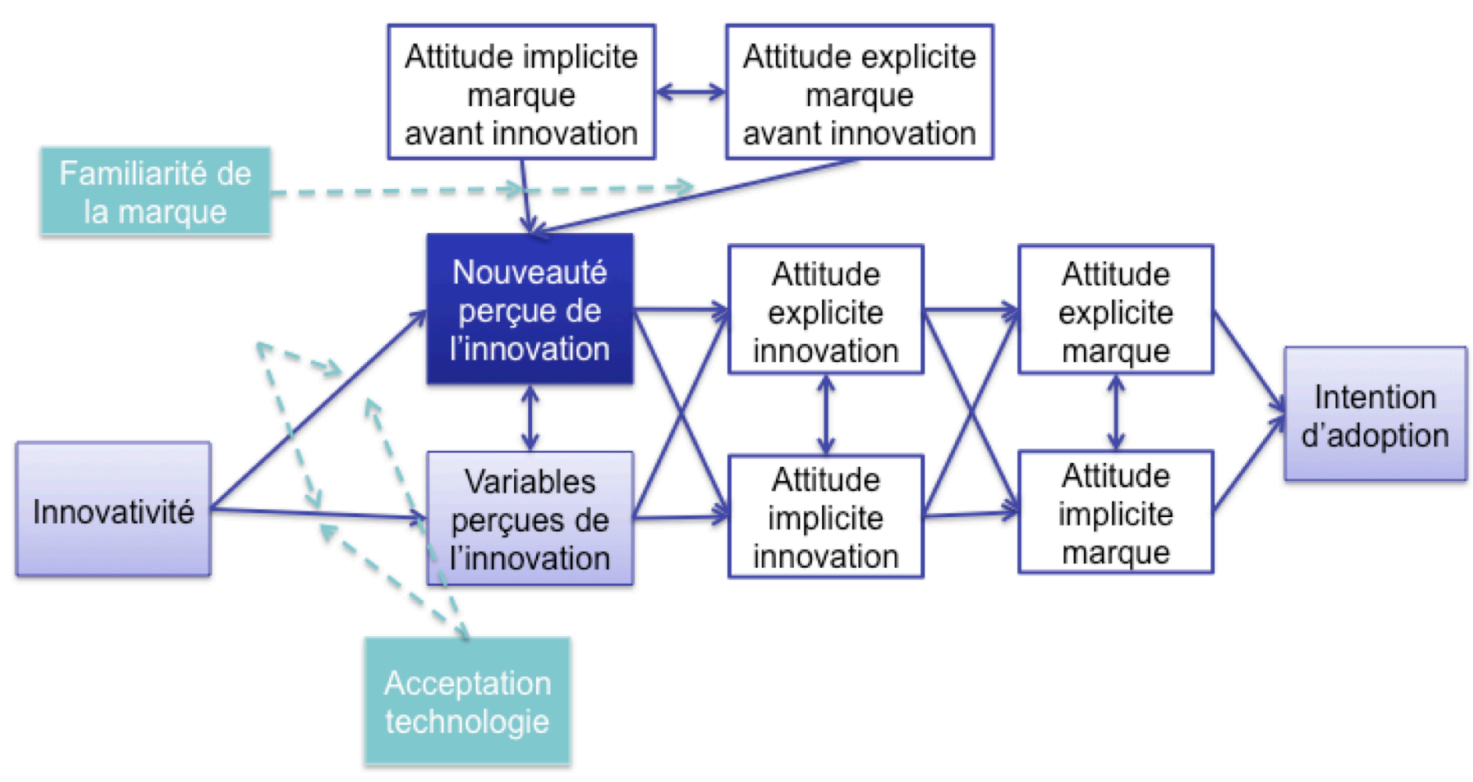

Figure 4 : Modèle global.

Ce modèle permet de mettre en évidence la place centrale de la nouveauté perçue dans l'évaluation d'une innovation et d'une marque innovante. Ne pas l'étudier ou la prendre en compte dans l'analyse et la diffusion d'une innovation représente un risque important pour l'adoption et la diffusion des innovations d'une part, mais également sur la perception de la marque comme innovante. Une innovation dont la nouveauté n'est pas perçue par les consommateurs peut porter préjudice à leur perception de la marque comme innovante.

Le modèle présenté sera enrichi par la prise en compte de la familiarité de la marque par le consommateur comme modérateur de l'influence de son attitude sur la nouveauté perçue. La prise en compte de l'implication envers le produit permettra également de modérer les résultats obtenus sur l'influence de l'innovativité des consommateurs sur la nouveauté perçue et les variables perçues de l'innovation. Enfin, l'acceptation des nouvelles technologies est une variable modératrice importante à prendre en considération dans ce modèle centré sur les innovations technologiques.

\section{Conclusion}

La nouveauté perçue représente un risque important dans l'analyse de l'influence des innovations sur la perception d'une marque innovante. Dans ce sens, la modélisation des influences de la perception des innovations sur l'attitude et l'évaluation d'une marque innovante permettra de développer un champ de recherche encore peu étudié, celui de la marque innovante, mais également celui de la nouveauté perçue.

La méthodologie envisagée pour valider ce modèle de recherche est la mise en place d'un questionnaire, permettant d'évaluer les différentes variables à l'aide d'échelles validées dans la littérature. L'analyse sera enrichie d'un Test d'Association Implicite (TAI) pour prendre en compte les attitudes implicites des consommateurs et affiner le modèle. La marque et l'innovation envisagées pour cette étude appartiennent au domaine technologique et écologique. 
Le modèle proposé permet d'évaluer la perception de la nouveauté, notamment dans le domaine technologique. La nouveauté perçue est un concept qui concerne l'ensemble des innovations disponibles sur le marché et dans tous les domaines. Tester le modèle sur différents types d'innovations permettrait d'approfondir l'étude de la nouveauté selon le type de produit. Par ailleurs, les influences entre l'innovation et la marque restent étudiées de manière parcellaire alors que cette compréhension permettrait aux marketeurs d'optimiser la diffusion des innovations. La clarification des différentes variables présentes dans le processus permettra de mettre à jour des points de vigilances pour faciliter le travail des managers. La prise en compte de ces nouveaux domaines de recherche pourra être intégrée dans les stratégies marketing des entreprises afin de replacer la perception des produits par les consommateurs au centre de leurs études pour mieux prédire l'adoption des innovations et ses impacts sur leur marque. Enfin, l'étude de la perception de la nouveauté d'une innovation par les consommateurs permettra aux marketeurs de réduire le risque d'échec des nouveaux produits en intégrant les attentes des consommateurs à leur stratégie de lancement d'une innovation.

\section{Bibliographie}

Ackermann, C.L., (2010), "Cognition implicite et comportement du consommateur : application à deux marques de véhicules automobiles ", Thèse de doctorat en sciences de gestion, Université de Nantes Institut d'Economie et de Management, Nantes.

Ackermann, C. L., Mathieu, J. P., (2012), « Risque et lancement de produits nouveaux: l'apport de la cognition sociale implicite », Management \& Avenir, Vol. 7, N57, pp. 160-176.

Andreassen, T. W., (2000), « Antecedents to satisfaction with service recovery », European Journal of Marketing, Vol. 34, N 1/2, pp. 156-175.

Anselmsson, J., Johansson, U., (2009), « Retailer brands and the impact on innovativeness in the grocery market », Journal of Marketing Management, Vol. 34, N8, pp. 75-95.

Arcuri, L., Castelli, L., Galdi, S., Zogmaister, C., Amadori, A. (2008), « Predicting the vote: Implicit Attitudes as predictors of the future behavior of decided and undecided voters », Political Psychology, Vol. 29, N`3, pp. 369-387.

Barone, M. J., Jewell, R. D., (2013), « The innovator's license: a latitude to deviate from category norms », Journal of Marketing, Vol. 77, $\mathrm{N}^{\circ} 1$, pp. 120-134.

Bartels, J., Reinders, M. J., (2011), « Consumer innovativeness and its correlates: A propositional inventory for future research ", Journal of Business Research, Vol. 64, Nº6, pp. 601-609.

Beverland, M. B., Napoli, J., Farrelly, F., (2010), "Can All Brands Innovate in the Same Way? A Typology of Brand Position and Innovation Effort », Journal of Product Innovation Management, Vol. 27, $\mathrm{N}^{\circ} 1$, pp. 33-48.

Bodenhausen, G., Todd, A., (2010), Automatic Aspects of Judgement and Decision Making, Handbook of implicit social cognition, The Guildford Press, New York.

Brunel F. F., Tietje, B. C., Greenwald A. G., (2004), « Is the implicit association test a valid and valuable measure of implicit consumer social cognition? », Journal of consumer Psychology, Vol. 14 $\mathrm{N}^{\circ} 4$, pp. 385-404.

Cestre, G. (1996), " Diffusion et innovativité: définition, modélisation et mesure », Recherche et Applications en Marketing, Vol. 11, N¹, pp.69-88.

Courbet, D., Fourquet-Courbet, M. P., (2005), « Modèle et mesures de l'influence de la communication : nouvelles perspectives ouvertes par la psychologie sociale », Market Management, Vol. 5, pp. 7-26. 
Fazio, R. H., Chen, J. M., McDonel, E. C., Sherman, S. J., (1982), "Attitude accessibility, attitudebehavior consistency, and the strength of the object-evaluation association », Journal of experimental social psychology, Vol. 18, $\mathrm{N}^{\circ} 4$, pp. 339-357.

Fazio, R.H., Olson, M.A., (2003), « Implicit measures in social cognition research: their meaning and use », Annual Review of Psychology, Vol.54, pp. 297-327.

Flight, R., Allaway A. W., Kim, W., D'Souza, G. (2011), " A study of perceived innovation characteristics across cultures and stages of diffusion », Journal of Marketing Theory and Practice, Vol. 19, N¹, pp. 109-126.

Greenwald, A.G., Banaji, M. R., (1995), "Implicit social cognition: attitudes, self esteem and stereotypes », Psychological Bulletin, Vol. 102, Nº1, pp. 4-27.

Hetet, B., Moutot, J. M., (2012), «Le risque de l'implicite sur l'acceptation d'une innovation et sur l'attitude envers la marque : une recherche exploratoire », Management et Avenir, Vol. 7, N57, pp. 177-194.

Hetet, B., Moutot, J. M., Mathieu, J. P., (2013), « Le risque de l'implicite dans le discours : le cas d'une innovation en faveur de l'écologie », Innovations, Vol. 1, N40, pp. 65-81.

Hirschman, E. C., (1980), "Innovativeness, novelty seeking, and consumer creativity ", Journal of Consumer Research, Vol. 7, N³, pp. 283-295.

Kaplan, M. D., (2009), " The relationship between perceived innovativeness and emotional product responses: a brand oriented approach », Innovative Marketing, Vol. 5, Nº1, pp. 42-50.

Liao, S., Cheng, C. C., (2012), «Brand equity and the exacerbating factors of product innovation failure evaluations: A communication effect perspective», Journal of Business Research, Vol. 67, $\mathrm{N}^{\circ} 1$, pp. 2919-2925.

Madhavaram, S., Appan, R., (2010), « The Potential Implications of Web-Based Marketing Communications for Consumers' Implicit and Explicit Brand Attitudes: A Call for Research », Psychology and Marketing, Vol. 27, N², pp. 186-202.

Maison, D., Greenwald, A.G., Bruin, R.H., (2004), « Predictive validity of the implicit association test studies of brands, consumer attitudes, and behavior », Journal of Consumer Psychology, Vol. 14, N4, pp. 405-415.

Meuter, M. L., Bitner, M. J., Ostrom A. L., Brown, S. W., (2005), « Choosing among alternative service delivery modes: an investigation of customer trial of self-service technologies », Journal of Marketing, Vol. 69, N², pp. 61-83.

Midgley, D. F., Dowling, G. R., (1978), « Innovativeness: the concept and its measurement » Journal of consumer research, Vol. 4, $\mathrm{N}^{\circ} 4$, pp. 229-242.

Nosek, B.A., (2007), « Understanding the individual implicity and explicity », International Journal of Psychology, Vol. 42, N³, pp. 184-188.

Olson, M.A., Fazio, R.H., (2003), " Relations between implicit measures of prejudice: what are we measuring? », Psychological Science, Vol. 14, №6, pp. 636-639.

Pauwels, K., Silva-Risso, J., Srinivasan, S., Hanssens, D. M. (2004), « New products, sales promotions, and firm value: The case of the automobile industry », Journal of marketing, Vol. 68, $\mathrm{N}^{\circ} 4$, pp. 142-156.

Perugini, M., Richetin, J., Zogmaister, C., (2010), Prediction of Behavior, Handbook of implicit social cognition, The Guildford Press, New York.

Raju, P. S., (1980), "Optimum stimulation level: its relationship to personality, demographics, and exploratory behavior », Journal of Consumer Research, Vol. 7, N³, pp. 272-282.

Rogers, E., (1962), The Diffusion of Innovations, The Free Press of Glencoe, New York.

Rogers, E., (2003), The Diffusion of Innovations : fourth edition, The Free Press, New York. 
Rogers, E., Shoemaker, F. F., (1971), Communication of innovations: a cross-cultural approach, The Free Press, New York.

Roehm, M. L., Brady, M. K., (2007), « Consumer Responses to Performance Failures by High Equity Brands », Journal of Consumer Research, Vol. 34, N4, pp. 537-545.

Roehrich, G., (1993), «Nouveauté perçue d'une innovation », Recherche et applications en Marketing, Vol. 9, N², pp. 19-41.

Roehrich, G., (2004), " Consumer innovativeness concept and measurement », Journal of Business Research, Vol. 57, N¹, pp. 671-677.

Schmitt, B., (2012), « The consumer psychology of brands », Journal of Consumer Psychology, Vol. 22, $\mathrm{N}^{\circ} 1$, pp. 7-17.

Smith, A. K., Bolton, R. N. (2002), « The effect of customers' emotional responses to service failures on their recovery effort evaluations and satisfaction judgments ", Journal of the Academy of Marketing Science, Vol. 30, Nº1, pp. 5-23.

Srinivasan, S., Pauwels, K., Silva-Risso, J., Hanssens, D. M. (2009), « Product innovations, advertising, and stock returns », Journal of Marketing, Vol. 73, N¹, pp. 24-43.

Staake, T., Thiesse, F., Fleisch, E., (2009), « The emergence of counterfeit trade: a literature review», European Journal of Marketing, Vol. 43, N³/4, pp. 320-349.

Steenkamp, J. B. E., Hofstede, F. T., Wedel, M., (1999), « A cross-national investigation into the individual and national cultural antecedents of consumer innovativeness », The Journal of Marketing, Vol. 63, N², pp. 55-69.

Steyer A., Zimmermann J. B., (2004), "Influence sociale et diffusion de l'innovation ", Mathematics and Social Sciences, Vol. 4, $\mathrm{N}^{\circ} 168$, pp. 43-57.

Tan, M., Teo, T., (2000), "Factors influencing the adoption of Internet Banking ", Journal of the Association for Information Sciences, Vol. 1, N¹, pp. 1-42.

Tellis, G., Yin, E., Bell, S., (2009), « Global consumer innovativeness: cross-country differences and demographic commonalities », Journal of International Marketing, Vol. 17, N², pp. 1-22.

Trendel, O., Warlop, L., (2005), « Présentation et applications des mesures implicites de restitution mémorielle en marketing », Recherches et Applications en Marketing, Vol. 20, N², pp. 77104.

Vandecasteele, B., Geuens, M., (2010), « Motived Consumer Innovativeness: Concept, Measurement and Validation », International Journal of Research in Marketing, Vol. 27, N44, pp. 308-318.

Venkatraman, M. P., Price, L. L., (1990), « Differentiating between cognitive and sensory innovativeness: concepts, measurement, and implications », Journal of Business Research, Vol. 20, N4, pp. 293-315.

Volle, P., (1995), « Le concept de risque perçu en psychologie du consommateur : antécédents et statut théorique », Recherche et Applications en Marketing, Vol. 10, N¹, pp. 39-56.

Wanke M., Plessner, H., Gartner T., (2002), « Mesuring Implicit Consumer Attitudes and Predicting Brand Choice », Advances in Consumer Research, Vol. 29, N¹, pp. 222.

Wells, J. D., Campbell, D. E., Valacich, J. S., Featherman, M., (2010), « The effect of perceived novelty on the adoption of information technology innovations: a risk/reward perspective ", Decision Science, Vol. 41, N4, pp. 813-843.

Zhuang, L., (1995), « Bridging the Gap Between Technology and Business Strategy: A Pilot Study on the Innovation Process », Management Decision, Vol. 33, Nº, pp. 13-21.

Ziamou, P. L., Ratneshwar, S., (2002), « Promoting consumer adoption of high-technology products: Is more information always better? ", Journal of Consumer Psychology, Vol. 12, N4, pp. 341351. 